\title{
BMJ Open Assessment of humoral and cell- mediated immune responses to pertussis vaccination: a systematic review protocol
}

\author{
Funbi Akinola, ${ }^{1,2}$ Rudzani Muloiwa, ${ }^{2,3}$ Gregory, D Hussey, ${ }^{2,4}$ Violette Dirix, ${ }^{5}$ \\ Benjamin Kagina, ${ }^{2}$ Edina Amponsah-Dacosta ${ }^{2}$
}

To cite: Akinola F, Muloiwa R, Hussey G,D, et al. Assessment of humoral and cell-mediated immune responses to pertussis vaccination: a systematic review protocol. BMJ Open 2019;9:e028109. doi:10.1136/ bmjopen-2018-028109

- Prepublication history and additional material for this paper are available online. To view these files, please visit the journal online (http://dx.doi. org/10.1136/bmjopen-2018028109).

Received 22 November 2018 Revised 23 April 2019 Accepted 17 May 2019
D) Check for updates

(C) Author(s) (or their employer(s)) 2019. Re-use permitted under CC BY-NC. No commercial re-use. See rights and permissions. Published by BMJ.

For numbered affiliations see end of article.

\section{Correspondence to}

Dr. Edina Amponsah-Dacosta; edina.amponsah-dacosta@uct. ac.za

\section{ABSTRACT}

Introduction Globally, some studies show a resurgence of pertussis. The risks and benefits of using whole-cell pertussis (wP) or acellular pertussis (aP) vaccines in the control of the disease have been widely debated. Better control of pertussis will require improved understanding of the immune response to pertussis vaccines. Improved understanding and assessment of the immunity induced by pertussis vaccines is thus imperative. Several studies have documented different immunological outcomes to pertussis vaccination from an array of assays. We propose to conduct a systematic review of the different immunological assays and outcomes used in the assessment of the humoraland cell-mediated immune response following pertussis vaccination.

Methods and analysis The primary outcomes for consideration are quality and quantity of immune responses (humoral and cell-mediated) post-pertussis vaccination. Of interest as secondary outcomes are types of immunoassays used in assessing immune responses post-pertussis vaccination, types of biological samples used in assessing immune responses post-pertussis vaccination, as well as the types of antigens used to stimulate these samples during post-pertussis vaccination immune response assessments. Different electronic databases (including PubMed, Cochrane, EBSCO Host, Scopus and Web of Science) will be accessed for peer-reviewed published and grey literature evaluating immune responses to pertussis vaccines between 1990 and 2019. The quality of included articles will be assessed using standardised risk and quality assessment tools specific to the study design used in each article. Data extraction will be done using a data extraction form. The extracted data will be analysed using STATA V.14.0 and RevMan V.5.3 software. A subgroup analysis will be conducted based on the study population, type of vaccine (wP or aP) and type of immune response (cellmediated or humoral). Guidelines for reporting systematic reviews in the revised 2009 Preferred Reporting Items for Systematic Reviews and Meta-Analysis (PRISMA) statement will be used in this study.

Ethics and dissemination Ethics approval is not required for this study as it is a systematic review. We will only make use of data already available in the public space. Findings will be reported via publication in a peerreviewed journal and presented at scientific meetings and workshops.

\section{Strengths and limitations of this study}

- The proposed systematic review will incorporate and synthesise evidence from a broad scope of study designs.

- Conclusions drawn from this work will extensively sum up strategic perspectives pertinent to evaluation of immune responses to pertussis vaccines.

- The findings will contribute to expanding the knowledge-base on the immune response to pertussis vaccines.

- Heterogeneity due to variations in the antigen formulations of acellular pertussis vaccines may limit the ability to conduct meta-analysis.

- The study will not review evidence from pre-clinical and in vitro studies.

Trial registration number CRD42018102455.

\section{INTRODUCTION}

\section{Background}

Pertussis or 'whooping cough' is a severe and highly contagious respiratory disease caused by the Gram-negative bacteria, Bordetella pertussis. ${ }^{1}$ Fortunately, pertussis is a vaccine preventable disease. There are two types of vaccines recommended for use against pertussis, the whole-cell pertussis $(\mathrm{wP})$ vaccine and the acellular pertussis $(\mathrm{aP})$ vaccine. Despite the availability of these vaccines, pertussis remains endemic worldwide. ${ }^{1}$ Although both vaccines have been shown to be safe and efficacious, neither vaccine provides long-lasting or lifelong immune protection against pertussis. ${ }^{2}$ Efforts to extend the immune protection conferred by pertussis vaccines and to develop new and improved vaccines have faced several challenges. One of these challenges is the fact that the immune response to pertussis vaccines is yet to be fully understood. ${ }^{23}$ What has been established is that both $\mathrm{aP}$ and $\mathrm{wP}$ 
induce humoral and cell-mediated immune responses. The humoral and cellular immune responses following pertussis vaccination have been characterised previously. ${ }^{4-6}$ However, systematic synthesis of the immunological assays and outcomes used to characterise the immune response to pertussis vaccines is lacking.

The humoral immune response commonly measured following pertussis vaccination include antibodies that are specific to purified $B$. pertussis antigens contained in the vaccine. Pertussis vaccines, produced by different manufacturers, contain varying formulations of these antigens. The aP antigens, for example, include purified pertussis toxin (PT), filamentous haemagglutinin (FHA) and pertactin (PRN). ${ }^{7}$ In addition to these three antigens, some aP may also contain a purified formulation of one or both serologically distinct fimbriae (FIM2/3). ${ }^{8}$ Measurement of the antibodies to these antigens can be quantitative (titres) or qualitative (avidity and subclasses). ${ }^{910}$ The commonly measured antibodies are IgG, and in some cases IgA. ${ }^{11} 12$ Following pertussis vaccination, IgG is the most frequent antibody isotype demonstrated in blood. ${ }^{10}{ }^{11}$ It has been established that all four major IgG subclasses (IgG1, IgG2, IgG3 and IgG4) are produced in response to PT, FHA and PRN antigens. ${ }^{10}$ In the absence of universally established correlates of immune protection against pertussis, measuring the distribution of these IgG subclasses to each of the vaccine antigens provides insight into protective immune response after pertussis vaccination. ${ }^{10}$ Overall, IgG1 has been shown to be the predominant IgG subclass produced for all pertussis antigens and in response to both $\mathrm{wP}$ and $\mathrm{aP}^{1013}$ In contrast, IgG2 and IgG3 generally contribute minimally to the total IgG response. ${ }^{10}$ An elevated IgG4 response has been reported among individuals primed with aP during infancy and in response to repeated aP booster vaccination. The elevated IgG4 response has been attributed to the T-helper 2 (Th2)-skewed immune response induced by $\mathrm{aP}^{10}{ }^{13}$ Detection of IgA is typically indicative of a prior infection but has also been demonstrated following pertussis vaccination. The IgA response has been reported to be significantly higher in response to $\mathrm{wP}$ compared with aP vaccines. ${ }^{12}$ The ELISA, Chinese Hamster Ovary (CHO) cell-based PT-neutralising antibody assay, as well as multiplex bead-based antibody assays are routinely used to measure the humoral immune response following pertussis vaccination. ${ }^{14-16}$ Another assay used in assessing the humoral immune response following vaccination with $\mathrm{wP}$ is the FIM agglutination assay. ${ }^{17}$ Serum and plasma are ideal biological samples to use for the measurement of specific antibodies induced following pertussis vaccination. ${ }^{13} 1819$

The cellular immune response to pertussis vaccines is mediated by Th cells, like the Th1, Th2 or Th $17 .{ }^{1820}$ While wP induces predominantly $\mathrm{Th} 1$ responses, aP induces a mixed Th2 and Th17 response. ${ }^{21-23}$ Pertussis-specific B-cell memory responses following vaccination have also been documented. ${ }^{25192425}$ Quantitative (frequencies of memory $\mathrm{T}$ cells and $\mathrm{B}$ cells and proportions of cytokine productions) and qualitative (multifunctional cytokine production) measurements of the pertussis-specific cell-mediated immune response can be characterised by flow cytometry. Other common assays used to assess the cell-mediated immune response include the multiplex bead-based immunoassay for cytokines, the enzyme-linked immunospot and the Fluorospot which count the number of cytokine-secreting cells (including specific immunoglobulin-producing B cells, antigen-specific plasma blasts and memory B cells) and measures IgG subclasses. ${ }^{1426}$ To evaluate cell-mediated immune responses to pertussis vaccines, the commonly used biological samples are whole blood and peripheral blood mononuclear cells. ${ }^{513}$

\section{Rationale}

Comprehensive characterisation of the immune response induced by pertussis vaccines remains a key focus area in the field of pertussis vaccinology. Recent reports of resurgence in the burden of pertussis in settings using either $\mathrm{wP}$ or aP have further driven the need to better understand the characteristics of the immune protection conferred by pertussis vaccines. ${ }^{27-30}$ The proposed systematic review will comprehensively synthesise the existing evidence on the humoral and cell-mediated immune response to $\mathrm{wP}$ and aP. For this purpose, we will synthesise and summarise evidence on the various assays used, the biological samples typically tested, the types of antigens used to stimulate these samples and the different immunological outcomes assessed. The primary intent of the review, therefore, will be to advance our limited understanding of the humoral and cell-mediated immune response induced after vaccination with either $\mathrm{wP}$ or aP.

\section{METHODS \\ Objective}

To carry out a systematic review and meta-analysis evaluating pertussis vaccine-induced humoral and cell-mediated immune responses.

\section{Primary objective}

- To describe the pertussis vaccine-induced humoral and cell-mediated immune responses following vaccination with $\mathrm{wP}$ and $\mathrm{aP}$.

\section{Secondary objectives}

- To describe the different biological samples used in assessing pertussis vaccine-induced immune responses.

- To describe the types of antigens used to stimulate samples during post-pertussis vaccination immune response assessments.

- To determine the array of immunological assays used in assessing pertussis vaccine-induced immune responses. 


\section{Eligibility criteria}

\section{Types of participants}

The proposed systematic review will incorporate studies involving newborns, infants, children, adolescents, and adults, and report on the humoral and cell-mediated immune responses following pertussis vaccination.

\section{Inclusion criteria}

- Studies that measure and report immune responses specific to pertussis vaccination in humans.

- Cross-sectional, case-control, prospective studies, randomised and non-randomised clinical trials, surveillance, before-and-after, interrupted time series and case-series study designs.

- Studies published between 1990 (the year of aP introduction) and 2018.

- Studies published in English.

\section{Exclusion criteria}

- Studies on infants in relation to perinatally transmitted pertussis-specific immunity.

- Pre-clinical and in vitro studies.

- Reviews including systematic reviews and meta-analysis.

- Studies published before 1990.

- Studies published in languages other than English.

Type of intervention

$\mathrm{aP}$ and $\mathrm{wP}$ vaccines.

\section{Outcomes}

\section{Primary outcomes}

- Quantity of immune responses (humoral and cell-mediated) post-pertussis vaccination.

- Quality of immune responses (humoral and cell-mediated) post-pertussis vaccination.

Secondary outcomes

- Types of immunoassays used in assessing immune responses post-pertussis vaccination.

- Types of antigens used to stimulate samples during post-pertussis vaccination immune response assessments.

- Types of biological samples used in assessing immune responses post-pertussis vaccination.

\section{Types of studies}

The proposed systematic review will include studies with the following designs: cross-sectional, case-control, prospective, randomised and non-randomised clinical trials, surveillance, before-and-after, interrupted time series and case-series. Retrospective and prospective studies, inpatient and outpatient as well as population-based studies will be included if they are of the requisite design.

\section{Study start and end dates}

The proposed systematic review is expected to begin in December 2018 and will be concluded by October 2019 .

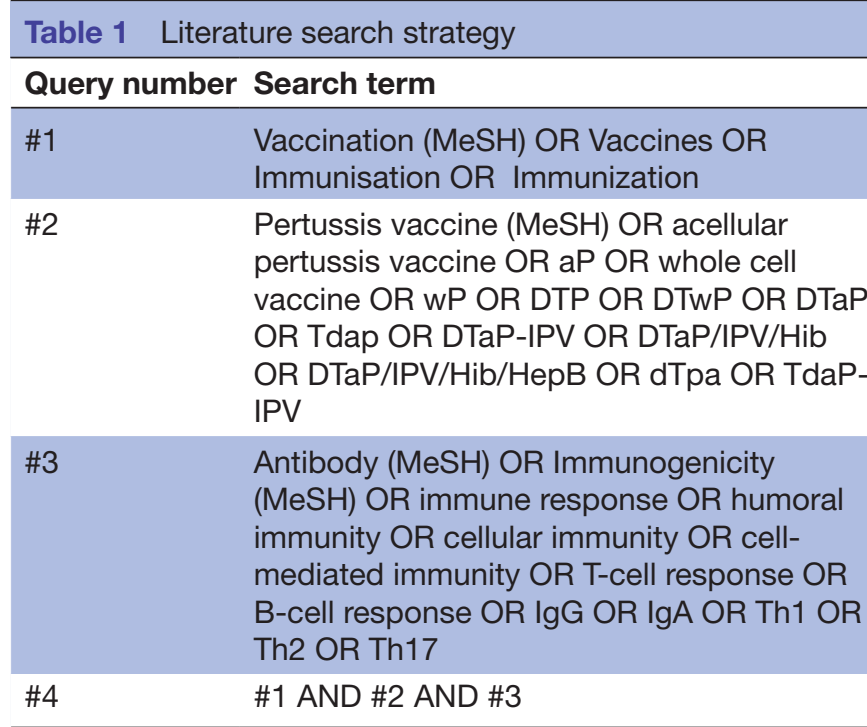

$\mathrm{MeSH}$, medical subject headings.

\section{Search strategy}

A rigorous search strategy will be used to identify studies published between January 1990 and April 2019. Keywords, search terms and associated synonyms relating to pertussis vaccination in different population groups will be used to retrieve the relevant literature. Medical subject headings as well as common terms will be used as appropriate. An attempt will be made to standardise the search strategy across all electronic databases and websites. Where database or website specifications do not make this possible, the search strategy will be suitably adapted. The search strategy developed for the review is presented in table 1 .

\section{Electronic databases}

The following electronic databases will be accessed during the literature search for published articles: PubMed, Cochrane, EBSCO Host, Scopus and Web of Science.

\section{Selection of eligible studies}

The first author (FA) will perform an initial screening of titles and abstracts to determine the eligibility of literature sources. Thereafter, full texts of studies meeting the inclusion criteria will be retrieved for a more detailed screening. Two independent reviewers (FA and EAD) will then read the full text of the studies to evaluate if they meet the inclusion criteria. Date of study publication, setting, design and methods, participants/groups and outcomes will be used as a guide when screening the full texts. Any discrepancies will be resolved by discussion and consensus with assistance from other co-reviewers.

\section{Data collection process}

A data extraction form will be used to collect relevant information from the studies selected for inclusion in the review. Where the required data are not clearly defined, an attempt will be made to contact the relevant authors for clarity. A template of the data extraction form is 
provided in the supplementary material Online supplementary table 1 shows the variables to be abstracted and these include the following:

- Study characteristics: study objectives, type of pertussis vaccine (vaccine name, brand, composition, details of chemical detoxification), vaccination schedule/ regimen used.

- Participant information: participants' demographic data, sample size, recruitment method.

- Methods: study design, inclusion criteria, antigens present in vaccines.

- Outcome measures: pertussis vaccine-induced immune response, immunoassays, antigens used during immunoassay assessments and biological samples tested.

In non-randomised control trials, the impact of the vaccination schedule on immune responses may be difficult to assess. However, as this study will primarily be assessing the quality and quantity of immune responses, we are of the opinion that the impact will be minimal, as this is more likely to be influenced by the period between receiving the last vaccine dose and the time of assessing the immune response than the number of previous doses received or the vaccination schedule adopted.

\section{Risk of bias in individual studies}

Eligible studies will be appraised using risk of bias tools based on the study design. In the case of cross-sectional studies, a modified risk of bias and quality of prevalence studies assessment tool described by Hoy $e t a l^{11}$ will be used (see online supplementary table 2). Bias reporting for the individual studies will be categorised as high risk, moderate risk and low risk based on aggregated scores.

\section{Data synthesis}

Quantitative data will be summarised and reported as proportions, mean or median estimates. Heterogeneity will be evaluated based on the age of study participants, vaccine type, immunological assays, immunological outcomes, type of biological samples tested, antigens used in the immunoassays and the study settings. Variations in study characteristics will be reported in a narrative manner. Quantitative data will be presented using tables while qualitative data will be described.

Statistical heterogeneity between study results will be evaluated using $\chi^{2}$ test of homogeneity, with an $\mathrm{I}^{2}$ statistic, where values of $25 \%$ reflect low heterogeneity, $50 \%$ medium heterogeneity and 75\% high heterogeneity. Data will be pooled where sufficient homogeneity is observed between evaluated studies ( $I^{2}$ statistic $\left.<50 \%\right)$. Furthermore, a funnel plot will be used to assess for publication bias. Finally, data analysis will be performed using STATA (STATA Corporation, College Station, TX, USA) V.14.0 for statistical calculations and RevMan V.5.3 for meta-analysis (Forest plots).

\section{Subgroup analysis}

A subgroup analysis will be conducted based on the study population, type of vaccine ( $\mathrm{wP}$ or $\mathrm{aP})$ and type of immune response (cell-mediated or humoral).

\section{Sensitivity analysis}

Sensitivity analysis will be used to determine the overall effect of study sample size (following exclusion of highly biased studies using the appropriate screening tool for each study type) on the study outcomes in the data that have been meta-analysed.

\section{Data management}

The first author will be in charge of data management and will from time to time collaborate with the last author $(\mathrm{EAD})$. An electronic main folder bearing the name of the project will be created. Other subdirectories will be created to store specific accessory tasks carried out vis-a-vis list of selected articles, compilation of articles included and excluded from the review, database of extracted information, risk of bias assessment outcomes, analysed data as well as working copies and completed review manuscripts. Three substitute copies of the main folder will be stored in two separate memory cards and the hard drive of a password-protected computer.

\section{Patient and public involvement}

Patients were not directly involved in defining the research question, or in the design and development of this systematic review protocol. The research focus for this protocol was defined by the authors by identifying a critical gap in the available evidence on pertussis vaccinology.

\section{Reporting the review}

The review protocol has been compiled using the PRISMA-P reporting guidelines. ${ }^{32}$ Guidelines for reporting systematic reviews in the revised 2009 Preferred Reporting Items for Systematic Reviews and MetaAnalyses (PRISMA) statement will be used in this study. An abridged study selection process using flow diagrams with a listing of excluded studies and why the studies were excluded will be used in the systematic review.

\section{DISCUSSION}

The reported resurgence of pertussis in countries using either $\mathrm{wP}$ or aP suggests the need to better understand the immune response to both vaccine types. ${ }^{30} 3334$ By better understanding the immune response to pertussis vaccines, it may be possible to develop evidence-based interventions such as optimal vaccination strategies or new and improved pertussis vaccines. Evidently, robust systematic evidence on the immune response to pertussis vaccines is needed to contribute to the field of pertussis vaccinology.

Furthermore, as the need and urgency to develop improved pertussis vaccines grows, so does the need to better characterise the immune response to currently recommended pertussis vaccines. It is anticipated that 
the findings of this systematic review will contribute to expanding the knowledge-base on the immune response to pertussis vaccines. Such evidence could inform global pertussis control efforts and guide the design of optimal studies aimed at assessing the immune response induced by pertussis vaccines.

\section{ETHICS AND DISSEMINATION}

Ethics approval is not required for this study as it is a systematic review. We will only make use of data already available in the public space. Findings will be reported via publication in a peer-reviewed journal and presented at scientific meetings and workshops.

\section{Author affiliations}

${ }^{1}$ Division of Epidemiology and Biostatistics, School of Public Health and Family

Medicine, University of Cape Town, Cape Town, South Africa

${ }^{2}$ Vaccines for Africa Initiative, School of Public Health and Family Medicine,

University of Cape Town, Cape Town, South Africa

${ }^{3}$ Department of Paediatrics and Child Health, Groote Schuur Hospital, University of Cape Town, Cape Town, South Africa

${ }^{4}$ Division of Medical Microbiology and Institute of Infectious Diseases and Molecular Medicine, University of Cape Town, Cape Town, South Africa

${ }^{5}$ Laboratory of Vaccinology and Mucosal Immunity, Université Libre de Bruxelles, Brussels, Belgium

Contributors This study was conceived by EAD, BMK, RM and GDH. FA developed the review protocol with input from all co-authors. FA will conduct the systematic review under the supervision of EAD and BMK. FA will also conduct the statistical analysis and data analysis for the systematic review. The literature search, screening and data extraction will be performed by FA, EAD and BMK. RM, VD and GDH will provide expert opinion and will review drafts of the systematic review.

Funding The authors have not declared a specific grant for this research from any funding agency in the public, commercial or not-for-profit sectors.

Competing interests None declared.

Patient consent for publication Not required.

Provenance and peer review Not commissioned; externally peer reviewed.

Open access This is an open access article distributed in accordance with the Creative Commons Attribution Non Commercial (CC BY-NC 4.0) license, which permits others to distribute, remix, adapt, build upon this work non-commercially, and license their derivative works on different terms, provided the original work is properly cited, appropriate credit is given, any changes made indicated, and the use is non-commercial. See: http://creativecommons.org/licenses/by-nc/4.0/.

\section{REFERENCES}

1. World Health Organization. Pertussis vaccine: WHO position paper. Wkly Epidemiol Rec 2015;90:433-60.

2. Edwards KM, Berbers GA. Immune responses to pertussis vaccines and disease. J Infect Dis 2014;209(Suppl.1):S10-5.

3. Locht $\mathrm{C}$. Will we have new pertussis vaccines? Vaccine 2018;36:5460-9.

4. Jefferson T, Rudin M, DiPietrantonj C. Systematic review of the effects of pertussis vaccines in children. Vaccine 2003;21(1718):2003-14.

5. Carollo M, Pandolfi E, Tozzi AE, et al. Humoral and B-cell memory responses in children five years after pertussis acellular vaccine priming. Vaccine 2014;32:2093-9.

6. Gröndahl-Yli-Hannuksela K, Kauko L, Van Der Meeren O, et al. Pertussis specific cell-mediated immune responses ten years after acellular pertussis booster vaccination in young adults. Vaccine 2016;34:341-9.

7. Agnolon V, Bruno C, Galletti B, et al. Multiplex immunoassay for in vitro characterization of acellular pertussis antigens in combination vaccines. Vaccine 2016;34:1040-6.
8. Gorringe AR, Vaughan TE. Bordetella pertussis fimbriae (Fim): relevance for vaccines. Expert Rev Vaccines 2014;13:1205-14.

9. Almanzar G, Ottensmeier B, Liese J, et al. Assessment of IgG avidity against pertussis toxin and filamentous hemagglutinin via an adapted enzyme-linked immunosorbent assay (ELISA) using ammonium thiocyanate. J Immunol Methods 2013;387:36-42.

10. Hendrikx LH, Schure RM, Oztürk K, et al. Different IgG-subclass distributions after whole-cell and acellular pertussis infant primary vaccinations in healthy and pertussis infected children. Vaccine 2011;29:6874-80.

11. Hendrikx LH, Berbers GA, Veenhoven RH, et al. IgG responses after booster vaccination with different pertussis vaccines in Dutch children 4 years of age: effect of vaccine antigen content. Vaccine 2009;27:6530-6.

12. Hendrikx LH, Öztürk K, de Rond LG, et al. Serum IgA responses against pertussis proteins in infected and Dutch WP or aP vaccinated children: an additional role in pertussis diagnostics. PLoS One 2011;6:e27681.

13. van der Lee S, Sanders EAM, Berbers GAM, et al. Whole-cell or acellular pertussis vaccination in infancy determines IgG subclass profiles to DTaP booster vaccination. Vaccine 2018;36:220-6.

14. Meade BD, Deforest A, Edwards KM, et al. Description and evaluation of serologic assays used in a multicenter trial of acellular pertussis vaccines. Pediatrics 1995;96(3 Pt 2):570-5.

15. Greco D, Salmaso S, Mastrantonio P, et al. A Controlled Trial of Two Acellular Vaccines and One Whole-Cell Vaccine against Pertussis. $N$ Engl J Med Overseas Ed 1996;334:341-9.

16. van der Zee A, Schellekens JF, Mooi FR. Laboratory Diagnosis of Pertussis. Clin Microbiol Rev 2015;28:1005-26.

17. Edwards KM, Meade BD, Decker MD, et al. Comparison of 13 acellular pertussis vaccines: overview and serologic response. Pediatrics 1995;96(3 Pt 2):548-57.

18. Schure RM, Hendrikx LH, de Rond LG, et al. T-cell responses before and after the fifth consecutive acellular pertussis vaccination in 4-year-old Dutch children. Clin Vaccine Immunol 2012;19:1879-86.

19. Jahnmatz M, Ljungman M, Netterlid E, et al. Pertussis-specific memory B-cell and humoral IgG responses in adolescents after a fifth consecutive dose of acellular pertussis vaccine. Clin Vaccine Immunol 2014;21:1301-8.

20. White OJ, Rowe J, Richmond P, et al. Th2-polarisation of cellular immune memory to neonatal pertussis vaccination. Vaccine 2010;28:2648-52.

21. Rowe J, Yerkovich ST, Richmond P, et al. Th2-associated local reactions to the acellular diphtheria-tetanus-pertussis vaccine in 4to 6-year-old children. Infect Immun 2005;73:8130-5.

22. Higgins SC, Jarnicki AG, Lavelle EC, et al. TLR4 mediates vaccineinduced protective cellular immunity to Bordetella pertussis: role of IL-17-producing T cells. J Immunol 2006;177:7980-9.

23. Vermeulen F, Verscheure V, Damis E, et al. Cellular immune responses of preterm infants after vaccination with whole-cell or acellular pertussis vaccines. Clin Vaccine Immunol 2010;17:258-62.

24. Hendrikx LH, Oztürk K, de Rond LG, et al. Identifying longterm memory B-cells in vaccinated children despite waning antibody levels specific for Bordetella pertussis proteins. Vaccine 2011;29:1431-7.

25. Hendrikx LH, de Rond LG, Oztürk K, et al. Impact of infant and preschool pertussis vaccinations on memory B-cell responses in children at 4 years of age. Vaccine 2011;29:5725-30.

26. Ausiello CM, Urbani F, la Sala A, La SA, et al. Vaccine- and antigendependent type 1 and type 2 cytokine induction after primary vaccination of infants with whole-cell or acellular pertussis vaccines. Infect Immun 1997;65:2168-74.

27. Cherry JD. The History of Pertussis (Whooping Cough); 19062015: facts, myths, and misconceptions. Curr Epidemiol Rep 2015;2:120-30

28. Güriș D, Strebel PM, Bardenheier B, et al. Changing epidemiology of pertussis in the United States: increasing reported incidence among adolescents and adults, 1990-1996. Clin Infect Dis 1999;28:1230-7.

29. Marchant CD, Loughlin AM, Lett SM, et al. Pertussis in Massachusetts, 1981-1991: incidence, serologic diagnosis, and vaccine effectiveness. $J$ Infect Dis 1994;169:1297-305.

30. Domenech de Cellès M, Magpantay FMG, King AA, et al. The impact of past vaccination coverage and immunity on pertussis resurgence. Sci Transl Med 2018;10:eaaj1748.

31. Hoy D, Brooks P, Woolf A, et al. Assessing risk of bias in prevalence studies: modification of an existing tool and evidence of interrater agreement. J Clin Epidemiol 2012;65:934-9.

32. Moher D, Shamseer L, Clarke M, et al. Preferred reporting items for systematic review and meta-analysis protocols (PRISMA-P) 2015 statement. Syst Rev 2015;4. 
33. Raguckas SE, VandenBussche HL, Jacobs C, et al. treatment, prevention, and beyond. Pharmacotherapy 2007;27:41-52.
34. Lapidot R, Gill CJ. The Pertussis resurgence: putting together the pieces of the puzzle. Trop Dis Travel Med Vaccines 2016;2:26. 